\title{
An alternating direction Galerkin method for a time-fractional partial differential equation with damping in two space dimensions
}

An Chen ${ }^{1}$ and Changpin $\mathrm{Li}^{2 *}$

"Correspondence: Icp@shu.edu.cn ${ }^{2}$ Department of Mathematics, Shanghai University, 99 Shangda Road, BaoShan District, Shanghai, 200444, China

Full list of author information is available at the end of the article

\begin{abstract}
In this paper, we propose an efficient alternating direction implicit (ADI) Galerkin method for solving the time-fractional partial differential equation with damping, where the fractional derivative is in the sense of Caputo with order in $(1,2)$. The presented numerical scheme is based on the $\mathrm{L}^{2-1} \sigma$ method in time and the Galerkin finite element method in space. The unconditional stability and convergence of the numerical scheme are both carefully proved. Numerical results are displayed for supporting the theoretical analysis.
\end{abstract}

Keywords: ADI Galerkin method; Caputo derivative; unconditional stability; convergence

\section{Introduction}

In this paper, we study the following time-fractional partial differential equation with damping in two space dimensions:

$$
\left\{\begin{array}{l}
{ }_{C} D_{0, t}^{\alpha} u+\lambda \frac{\partial u}{\partial t}=\Delta u+f(x, y, t), \quad(x, y, t) \in \Omega \times(0, T], T>0, \\
\left.u\right|_{\partial \Omega}=0, \quad(x, y, t) \in \partial \Omega \times(0, T], \\
\left.u\right|_{t=0}=u_{0}(x, y),\left.\quad \frac{\partial u}{\partial t}\right|_{t=0}=\varphi(x, y), \quad(x, y) \in \Omega,
\end{array}\right.
$$

where $1<\alpha<2, \lambda\rangle 0, \Delta$ is a Laplacian which characterizes the standard diffusion in space, $\Omega=(a, b) \times(c, d), f$ is the source term, and ${ }_{C} D_{0, t}^{\alpha}$ is the Caputo derivative operator which is defined by

$$
{ }_{C} D_{0, t}^{\alpha} g(t)={ }_{R L} D_{0, t}^{-(2-\alpha)}\left(g^{\prime \prime}(t)\right)=\frac{1}{\Gamma(2-\alpha)} \int_{0}^{t}(t-s)^{1-\alpha} g^{\prime \prime}(s) d s, \quad 1<\alpha<2,
$$

and ${ }_{R L} D_{0, t}^{-\beta}$ is the fractional integral operator defined by

$$
{ }_{R L} D_{0, t}^{-\beta} g(t)=\frac{1}{\Gamma(\beta)} \int_{0}^{t}(t-s)^{\beta-1} g(s) d s \quad \text { for } \beta>0 .
$$


We remark that there are also other different definitions for fractional derivative (see, for examples, $[1,2])$, here we only focus on the Caputo derivative.

Fractional partial differential equations arise in a wide variety of physical situations which are often used to demonstrate the anomalous diffusion behaviors, for instance, diffusion of plasma, transport of fluid in porous media, diffusion at liquid surfaces, Lévy flights in the two-dimensional rotating flow, etc. [3, 4]. Due to the nonlocal properties of a fractional differential operator, fractional partial differential equations have already been proved to be highly effective in depicting those anomalous diffusion scenarios. Equation (1) can be characterized as a wave equation with a damping effect due to the nonzero term $\lambda \frac{\partial u}{\partial t}$. It can be regarded as the fractional analogue of the classical telegraph equation (if $\alpha=2$, then it is just the classical telegraph equation). Choosing suitable $\lambda$, for example, $\lambda=1$, model (1) becomes a special case of the fractional Cattaneo-type equation [5]. When $\lambda=0$, it reduces to the single-term counterpart, i.e., the standard time-fractional partial differential equation

$$
{ }_{C} D_{0, t}^{\alpha} u=\Delta u+f \quad \text { in } \Omega \times(0, T] .
$$

It is known that, according to the order of the time-fractional derivative, the anomalous diffusions are divided into sub-diffusion $(\alpha \in(0,1))$ and super-diffusion or diffusion-wave $(\alpha \in(1,2))$ [3]. These anomalous diffusions are in the sense of time. Normal diffusion as used in space and anomalous diffusion as utilized in time may make confusion. So, we would rather call (2) time-fractional partial differential equation (or time-fractional hyperbolic equation) than time-fractional diffusion-wave equation.

Several approaches are available for solving time-fractional partial differential equations in one or more than one space dimension, see [6-13] for finite difference methods, [14-18] for finite element methods, and $[19,20]$ for spectral methods. Moreover, one can refer to the book [21] or the review paper [22] for more details. Due to the nonzero $\lambda$ in the damping term, the numerical approaches for (1) are different from those for (2). In [23], Chen et al. derived the analytical solution for equation (1) in one space dimension by using the method of separation of variables, then constructed an implicit difference approximation, where the stability and convergence were proved by the energy method. Very recently, Ren and Gao presented a compact ADI scheme for the two-dimensional fractional Cattaneo equation [24], i.e., letting $\lambda=1$ in (1). Zhao and Sun also considered a fractional version of the Cattaneo equation with variable coefficient [25]. They proposed the corresponding compact Crank-Nicolson scheme and compact Crank-Nicolson ADI scheme for the one and two space dimensions, respectively.

Although time-fractional partial differential equations involving the Caputo derivative operator describe the history-dependent behavior well, they also cause large computational cost due to the fact that all the previous numerical data need to be stored in order to obtain the current numerical solution. So, seeking proper numerical schemes for solving (1), such as these with high accuracy and less storage, becomes important. Thus, the most obvious thing is to propose an efficient high order ADI method for model (1). In this paper, we aim to construct an ADI Galerkin scheme for solving (1). By using the L2$1_{\sigma}$ method for the Caputo derivative and the ADI finite element method for the spatial derivative, we obtain an efficient ADI Galerkin scheme for solving (1). We also carefully display the corresponding theoretical analyses, such as stability and error estimate, for the 
derived scheme. It is worthy to mention that the proposed method is derived on the direct discretization for the Caputo derivative at the non-grid point in time which is different from those existing numerical methods, such as [15].

Throughout this paper, we assume that $f, u_{0}$ and $\varphi$ are suitably smooth functions, and the solution of problem (1) is unique and sufficiently regular. A brief outline of this paper is given as follows. In Section 2, we introduce the preliminaries. In Section 3, an ADI Galerkin scheme for solving time-fractional hyperbolic equation with damping is derived. The corresponding unconditional stability and convergence analysis are given in Section 4. In Section 5, numerical results are presented to support the theoretical analysis. Section 6 concludes the paper and presents future work.

\section{Preliminaries}

In the following, we always assume that $C$ denotes a generic positive constant which is independent of the mesh spacing.

Let $\tau=T / N$, where $N$ is a positive integer. Denote $t_{n}=n \tau, 0 \leq n \leq N$, and $t_{n+\frac{1}{2}}=$ $\left(t_{n+1}+t_{n}\right) / 2,0 \leq n \leq N-1$. The time domain $[0, T]$ is covered by $\Omega_{\tau}=\left\{t_{n} \mid 0 \leq n \leq N\right\}$. Given the mesh function $v=\left\{v^{n} \mid 0 \leq n \leq N\right\}$ on $\Omega_{\tau}$, denote

$$
\delta_{t} v^{n+\frac{1}{2}}=\frac{1}{\tau}\left(v^{n+1}-v^{n}\right), \quad v^{n+\frac{1}{2}}=\frac{1}{2}\left(v^{n+1}+v^{n}\right) .
$$

We firstly give the discrete scheme of the Caputo derivative. Differing from the classical discrete scheme for Caputo derivative at $t=t_{n}$, the L2-1 $\sigma$ scheme approximates the Caputo derivative at $t=t_{n+\sigma}$, and it will be used in this paper. That is, for $g(t) \in C^{3}[0, T]$, the L2-1 scheme is described as follows [26]:

$$
\left.{ }_{C} D_{0, t}^{\beta} g(t)\right|_{t=n+\sigma}=\frac{1}{\hat{\mu}} \sum_{k=0}^{n} c_{n-k}^{(n+1)}\left[g\left(t_{k+1}\right)-g\left(t_{k}\right)\right]+R_{1}^{n+\sigma},
$$

where $\beta \in(0,1)$, the truncation error

$$
\left|R_{1}^{n+\sigma}\right| \leq C \tau^{3-\beta} \max _{0 \leq t \leq T}\left|g^{\prime \prime \prime}(t)\right|
$$

the coefficients $\hat{\mu}=\Gamma(2-\beta) \tau^{\beta}, \sigma=1-\beta / 2$, and for $n=0, c_{0}^{(1)}=a_{0}$, for $n \geq 1$,

$$
c_{k}^{(n+1)}= \begin{cases}\hat{a}_{0}+\hat{b}_{1}, & k=0, \\ \hat{a}_{k}+\hat{b}_{k+1}-\hat{b}_{k}, & k=1,2, \ldots, n-1, \\ \hat{a}_{n}-\hat{b}_{n}, & k=n .\end{cases}
$$

Here, $\hat{a}_{0}=\sigma^{1-\beta}, \hat{a}_{k}=(k+\sigma)^{1-\beta}-(k-1+\sigma)^{1-\beta}, k \geq 1$ and $\hat{b}_{k}=\frac{1}{2-\beta}\left[(k+\sigma)^{2-\beta}-\right.$ $\left.(k-1+\sigma)^{2-\beta}\right]-\frac{1}{2}\left[(k+\sigma)^{1-\beta}+(k-1+\sigma)^{1-\beta}\right], k \geq 1$.

We also have the following approximations to $d g\left(t_{n+\sigma}\right) / d t$ and $g\left(t_{n+\sigma}\right)$, respectively:

$$
\begin{aligned}
& \frac{d g\left(t_{n+\sigma}\right)}{d t}=\hat{\delta}_{t} g\left(t_{n}\right)+R_{2}^{n+\sigma}, \quad n \geq 1, \\
& g\left(t_{n+\sigma}\right)=g^{n+\sigma}+R_{3}^{n+\sigma}, \quad n \geq 0,
\end{aligned}
$$


where $\hat{\delta}_{t} g\left(t_{n}\right)=\frac{1}{2 \tau}\left[(2 \sigma+1) g\left(t_{n+1}\right)-4 \sigma g\left(t_{n}\right)+(2 \sigma-1) g\left(t_{n-1}\right)\right]$, and $g^{n+\sigma}=\sigma g\left(t_{n+1}\right)+$ $(1-\sigma) g\left(t_{n}\right)$. The truncation errors have the following estimate:

$$
\left|R_{2}^{n+\sigma}\right| \leq C \tau^{2} \max _{0 \leq t \leq T}\left|g^{\prime \prime \prime}(t)\right|, \quad\left|R_{3}^{n+\sigma}\right| \leq C \tau^{2} \max _{0 \leq t \leq T}\left|g^{\prime \prime}(t)\right|
$$

For the spatial direction, we recall some notations and properties from the finite element methods. We limit our interest to the two space dimensions.

For a nonnegative integer $m$, denote $H^{m}(\Omega)$ as the Sobolev space $W^{m, 2}(\Omega)$. Denote $H_{0}^{1}(\Omega)$ as the closure of $C_{0}^{\infty}(\Omega)$ with respect to the norm $\|\cdot\|_{H^{1}}$. An equivalent norm on $H_{0}^{1}(\Omega)$ is

$$
\|v\|_{H_{0}^{1}}=\|\nabla v\|=\left(\left\|\frac{\partial v}{\partial x}\right\|^{2}+\left\|\frac{\partial v}{\partial y}\right\|^{2}\right)^{1 / 2}
$$

where $\|\cdot\|$ denotes the $L^{2}$ norm.

Denote

$$
Z=\left\{u \mid u, \frac{\partial u}{\partial x}, \frac{\partial u}{\partial y}, \frac{\partial^{2} u}{\partial x \partial y} \in L^{2}(\Omega)\right\}
$$

For $h>0$ and $r \geq 1, X_{h}^{r} \subset H_{0}^{1}(\Omega)$ is a finite-dimensional subspace consisting of all piecewise polynomials of degree at most $r$. And $X_{h}^{r}$ satisfies the following assumptions [27]:

$$
\left\{\begin{array}{l}
X_{h}^{r} \subset Z \cap H_{0}^{1}, \\
\left\|\frac{\partial^{2} \chi}{\partial x \partial y}\right\| \leq C h^{-2}\|\chi\| \quad \text { for } \chi \in X_{h}^{r}, \\
\inf _{\chi \in X_{h}^{r}}\left[\sum_{m=0}^{2} h^{m} \sum_{\substack{i, j=0,1 \\
i+j=m}}\left\|\frac{\partial^{m}(u-x)}{\partial x^{i} \partial y^{j}}\right\|\right] \leq C h^{s}\|u\|_{H^{s}} \\
\quad \text { for } u \in H^{s}(\Omega) \cap Z \cap H_{0}^{1}(\Omega), 2 \leq s \leq r+1, s \in Z^{+} .
\end{array}\right.
$$

\section{Derivation of the ADI Galerkin scheme}

Note that ${ }_{C} D_{0, t}^{\alpha} u={ }_{C} D_{0, t}^{\beta}(\partial u / \partial t), \beta=\alpha-1$, so we rewrite equation (1) as a first-order system by setting $\phi=\partial u / \partial t$. That is,

$$
\left\{\begin{array}{l}
{ }_{C} D_{0, t}^{\beta} \phi+\lambda \phi=\Delta u+f(x, y, t), \quad(x, y, t) \in \Omega \times(0, T], T>0, \\
\frac{\partial u}{\partial t}=\phi .
\end{array}\right.
$$

Now considering (8) on the time level $t_{n+\sigma}$ for $n \geq 0\left(t_{1 / 2}\right.$ when $n=0$ for the second equation of (8)) and using the approximations (3) and (6), we get

$$
\left\{\begin{array}{l}
\frac{1}{\hat{\mu}} \sum_{k=0}^{n} c_{n-k}^{(n+1)}\left(\phi^{k+1}-\phi^{k}\right)+\lambda \phi^{n+\sigma}=\Delta u^{n+\sigma}+f^{n+\sigma}+O\left(\tau^{2}\right), \\
\delta_{t} u^{1 / 2}=\phi^{1 / 2}+O\left(\tau^{2}\right), \\
\hat{\delta}_{t} u^{n}=\phi^{n+\sigma}+O\left(\tau^{2}\right) .
\end{array}\right.
$$

Note that $u^{1}=u^{0}+\tau\left(\phi^{1}+\phi^{0}\right) / 2$ and

$$
u^{n+1}=\frac{2 \tau}{2 \sigma+1}\left[\sigma \phi^{n+1}+(1-\sigma) \phi^{n}\right]+\frac{1}{2 \sigma+1}\left[4 \sigma u^{n}-(2 \sigma-1) u^{n-1}\right]
$$


for the second and third equations in (9). Substituting them into the first equation in (9) for the two cases $n=0$ and $n \geq 1$, one has

$$
\begin{aligned}
\phi^{1} & -\sigma \frac{\tau}{2} \frac{\hat{\mu}}{c_{0}^{(1)}+\lambda \hat{\mu} \sigma} \Delta \phi^{1} \\
& =\frac{1}{c_{0}^{(1)}+\lambda \hat{\mu} \sigma}\left[\left(c_{0}^{(1)}-\lambda \hat{\mu}(1-\sigma)\right) \phi^{0}+\sigma \hat{\mu} \frac{\tau}{2} \Delta \phi^{0}+\hat{\mu} \Delta u^{0}+\hat{\mu} f^{\sigma}\right]
\end{aligned}
$$

and

$$
\begin{aligned}
\phi^{n+1} & -\frac{2 \tau}{2 \sigma+1} \frac{\sigma^{2} \hat{\mu}}{c_{0}^{(n+1)}+\lambda \hat{\mu} \sigma} \Delta \phi^{n+1} \\
= & \frac{1}{c_{0}^{(n+1)}+\lambda \hat{\mu} \sigma}\left[-\sum_{k=0}^{n-1} c_{n-k}^{(n+1)}\left(\phi^{k+1}-\phi^{k}\right)\right. \\
& +\left(c_{0}^{(n+1)}-\lambda \hat{\mu}(1-\sigma)\right) \phi^{n}+2 \sigma(1-\sigma) \hat{\mu} \frac{\tau}{2 \sigma+1} \Delta \phi^{n} \\
& \left.+\left(\frac{4 \sigma^{2} \hat{\mu}}{2 \sigma+1}+(1-\sigma) \hat{\mu}\right) \Delta u^{n}-\frac{\sigma(2 \sigma-1) \hat{\mu}}{2 \sigma+1} \Delta u^{n-1}+\hat{\mu} f^{n+\sigma}\right], \quad n \geq 1 .
\end{aligned}
$$

It follows from (10) and (11) that the Galerkin approximation $\left\{\phi_{h}^{n}\right\}_{n=0}^{N} \subset X_{h}^{r}$ for solving (1) is defined by

$$
\begin{aligned}
\left(\phi_{h}^{1}, \chi\right)+\sigma \frac{\tau}{2} \frac{\hat{\mu}}{c_{0}^{(1)}+\lambda \hat{\mu} \sigma}\left(\nabla \phi_{h}^{1}, \nabla \chi\right) & \\
= & \frac{1}{c_{0}^{(1)}+\lambda \hat{\mu} \sigma}\left[\left(c_{0}^{(1)}-\lambda \hat{\mu}(1-\sigma)\right)\left(\phi_{h}^{0}, \chi\right)\right. \\
& \left.-\sigma \hat{\mu} \frac{\tau}{2}\left(\nabla \phi_{h}^{0}, \nabla \chi\right)-\hat{\mu}\left(\nabla u_{h}^{0}, \nabla \chi\right)+\hat{\mu}\left(f^{\sigma}, \chi\right)\right], \quad \forall \chi \in X_{h}^{r}, n=0,
\end{aligned}
$$

and

$$
\begin{aligned}
& \left(\phi_{h}^{n+1}, \chi\right)+\frac{2 \tau}{2 \sigma+1} \frac{\sigma^{2} \hat{\mu}}{c_{0}^{(n+1)}+\lambda \hat{\mu} \sigma}\left(\nabla \phi_{h}^{n+1}, \nabla \chi\right) \\
& =\frac{1}{c_{0}^{(n+1)}+\lambda \hat{\mu} \sigma}\left[-\sum_{k=0}^{n-1} c_{n-k}^{(n+1)}\left(\left(\phi_{h}^{k+1}-\phi^{k}\right), \chi\right)\right. \\
& \quad+\left(c_{0}^{(n+1)}-\lambda \hat{\mu}(1-\sigma)\right)\left(\phi_{h}^{n}, \chi\right)-2 \sigma(1-\sigma) \hat{\mu} \frac{\tau}{2 \sigma+1}\left(\nabla \phi_{h}^{n}, \nabla \chi\right) \\
& \quad-\left(\frac{4 \sigma^{2} \hat{\mu}}{2 \sigma+1}+(1-\sigma) \hat{\mu}\right)\left(\nabla u_{h}^{n}, \nabla \chi\right)+\frac{\sigma(2 \sigma-1) \hat{\mu}}{2 \sigma+1}\left(\nabla u_{h}^{n-1}, \nabla \chi\right) \\
& \left.\quad+\hat{\mu}\left(f^{n+\sigma}, \chi\right)\right], \quad \forall \chi \in X_{h}^{r}, n \geq 1 .
\end{aligned}
$$

Here, the initial values $u_{h}^{0}$ and $\varphi_{h}$ are given by

$$
\left(u_{h}^{0}-u_{0}, \chi\right)=0, \quad\left(\varphi_{h}-\varphi, \chi\right)=0, \quad \chi \in X_{h}^{r},
$$


respectively. From (12) and (13) we can obtain the approximation to $u$, namely, $u_{h}^{1}=u^{0}+$ $\tau\left(\phi_{h}^{1}+\phi_{h}^{0}\right) / 2$ for $n=0$ and

$$
u_{h}^{n+1}=\frac{2 \tau}{2 \sigma+1}\left[\sigma \phi_{h}^{n+1}+(1-\sigma) \phi_{h}^{n}\right]+\frac{1}{2 \sigma+1}\left[4 \sigma u_{h}^{n}-(2 \sigma-1) u_{h}^{n-1}\right] \quad \text { for } n \geq 1 .
$$

Adding the two small terms

$$
\begin{aligned}
& 2\left(\sigma \frac{\tau}{2} \frac{\hat{\mu}}{c_{0}^{(1)}+\lambda \hat{\mu} \sigma}\right)^{2}\left(\frac{\partial^{2}}{\partial x \partial y} \phi_{h}^{1 / 2}, \frac{\partial^{2}}{\partial x \partial y} \chi\right), \\
& \sigma^{3}\left(\frac{2 \tau}{2 \sigma+1} \frac{\hat{\mu}}{c_{0}^{(n+1)}+\lambda \hat{\mu} \sigma}\right)^{2}\left(\frac{\partial^{2}}{\partial x \partial y} \phi_{h}^{n+\sigma}, \frac{\partial^{2}}{\partial x \partial y} \chi\right)
\end{aligned}
$$

on the left-hand sides of (12) and (13), respectively, the ADI Galerkin scheme for (1) in the inner form is given below.

$$
\left\{\begin{array}{c}
\left(\phi_{h}^{1}, \chi\right)+\sigma \frac{\tau}{2} \frac{\hat{\mu}}{c_{0}^{(1)}+\lambda \hat{\mu} \sigma}\left(\nabla \phi_{h}^{1}, \nabla \chi\right)+2\left(\sigma \frac{\tau}{2} \frac{\hat{\mu}}{c_{0}^{(1)}+\lambda \hat{\mu} \sigma}\right)^{2}\left(\frac{\partial^{2}}{\partial x \partial y} \phi_{h}^{1 / 2}, \frac{\partial^{2}}{\partial x \partial y} \chi\right) \\
=\frac{1}{c_{0}^{(1)}+\lambda \hat{\mu} \sigma}\left[\left(c_{0}^{(1)}-\lambda \hat{\mu}(1-\sigma)\right)\left(\phi_{h}^{0}, \chi\right)\right. \\
\left.\quad-\sigma \hat{\mu} \frac{\tau}{2}\left(\nabla \phi_{h}^{0}, \nabla \chi\right)-\hat{\mu}\left(\nabla u_{h}^{0}, \nabla \chi\right)+\hat{\mu}\left(f^{\sigma}, \chi\right)\right], \quad \forall \chi \in X_{h}^{r}, n=0, \\
\left(\phi_{h}^{n+1}, \chi\right)+\frac{2 \tau}{2 \sigma+1} \frac{\sigma^{2} \hat{\mu}}{c_{0}^{(n+1)}+\lambda \hat{\mu} \sigma}\left(\nabla \phi_{h}^{n+1}, \nabla \chi\right)+\sigma^{3}\left(\frac{2 \tau}{2 \sigma+1} \frac{\hat{\mu}}{c_{0}^{(n+1)}+\lambda \hat{\mu} \sigma}\right)^{2}\left(\frac{\partial^{2} \phi_{h}^{n+\sigma}}{\partial x \partial y}, \frac{\partial^{2} \chi}{\partial x \partial y}\right) \\
=\frac{1}{c_{0}^{(n+1)}+\lambda \hat{\mu} \sigma}\left[-\sum_{k=0}^{n-1} c_{n-k}^{(n+1)}\left(\left(\phi_{h}^{k+1}-\phi^{k}\right), \chi\right)+\left(c_{0}^{(n+1)}-\lambda \hat{\mu}(1-\sigma)\right)\left(\phi_{h}^{n}, \chi\right)\right. \\
\quad-2 \sigma(1-\sigma) \hat{\mu} \frac{\tau}{2 \sigma+1}\left(\nabla \phi_{h}^{n}, \nabla \chi\right)-\left(\frac{4 \sigma^{2} \hat{\mu}}{2 \sigma+1}+(1-\sigma) \hat{\mu}\right)\left(\nabla u_{h}^{n}, \nabla \chi\right) \\
\left.\quad+\frac{\sigma(2 \sigma-1) \hat{\mu}}{2 \sigma+1}\left(\nabla u_{h}^{n+1}, \nabla \chi\right)+\hat{\mu}\left(f^{n+\sigma}, \chi\right)\right], \quad \forall \chi \in X_{h}^{r}, n \geq 1,
\end{array}\right.
$$

from which we can obtain the approximation to $u: u_{h}^{1}=u^{0}+\tau\left(\phi_{h}^{1}+\phi_{h}^{0}\right) / 2$ for $n=0$ and

$$
u_{h}^{n+1}=\frac{2 \tau}{2 \sigma+1}\left[\sigma \phi_{h}^{n+1}+(1-\sigma) \phi_{h}^{n}\right]+\frac{1}{2 \sigma+1}\left[4 \sigma u_{h}^{n}-(2 \sigma-1) u_{h}^{n-1}\right]
$$

for $n \geq 1$.

Next, we further discuss numerical scheme (14). In order to efficiently solve the numerical scheme (14), we rewrite it into a more familiar matrix form. Assume that $X_{h}^{r}=$ $X_{h, x}^{r} \otimes X_{h, y}^{r}$, where $X_{h, x}^{r}$ and $X_{h, y}^{r}$ are finite-dimensional subspaces of $H_{0}^{1}([a, b])$ and $H_{0}^{1}([c, d])$, respectively. Let $\left\{\varphi_{i} \theta_{j}\right\}_{i=1, j=1}^{N_{x}, N_{y}}$ be the tensor product basis of $X_{h}^{r}$, where $\left\{\varphi_{i}\right\}_{i=1}^{N_{x}}$ and $\left\{\theta_{j}\right\}_{j=1}^{N_{y}}$ are bases of $X_{h, x}^{r}$ and $X_{h, y}^{r}$, respectively. Let

$$
u_{h}^{n}(x, y)=\sum_{i=1}^{N_{x}} \sum_{j=1}^{N_{y}} \alpha_{i j}^{(n)} \varphi_{i}(x) \theta_{j}(y), \quad \phi_{h}^{n}(x, y)=\sum_{i=1}^{N_{x}} \sum_{j=1}^{N_{y}} \beta_{i j}^{(n)} \varphi_{i}(x) \theta_{j}(y),
$$

then $\alpha_{i j}^{(1)}=\alpha_{i j}^{(0)}+\tau\left(\beta_{i j}^{(1)}+\beta_{i j}^{(0)}\right) / 2$ for $n=0$ and

$$
\alpha_{i j}^{(n+1)}=\frac{2 \tau}{2 \sigma+1}\left[\sigma \beta_{i j}^{(n+1)}+(1-\sigma) \beta_{i j}^{(n)}\right]+\frac{1}{2 \sigma+1}\left[4 \sigma \alpha_{i j}^{(n)}-(2 \sigma-1) \alpha_{i j}^{(n-1)}\right]
$$


for $n \geq 1$. Set matrices $M_{x}, M_{y}, S_{x}, S_{y} \in \mathbb{R}^{N_{x} \times N_{y}}$ satisfying

$$
\begin{aligned}
& \left(M_{x}\right)_{i, j}=\left(\varphi_{i}, \varphi_{j}\right), \quad\left(M_{y}\right)_{i, j}=\left(\theta_{i}, \theta_{j}\right), \\
& \left(S_{x}\right)_{i, j}=\left(\frac{\partial \varphi_{i}}{\partial x}, \frac{\partial \varphi_{j}}{\partial x}\right), \quad\left(S_{y}\right)_{i, j}=\left(\frac{\partial \theta_{i}}{\partial x}, \frac{\partial \theta_{j}}{\partial x}\right) .
\end{aligned}
$$

Inserting $u_{h}^{n}$ and $\phi_{h}^{n}$ into (14) and choosing $\chi=\varphi_{k} \theta_{m}, k=1,2, \ldots, N_{x}, m=1,2, \ldots, N_{y}$, we obtain the matrix form of the ADI Galerkin scheme (14) as follows.

$$
\left\{\begin{array}{l}
{\left[M_{x}+\sigma \frac{\tau}{2} \frac{\hat{\mu}}{c_{0}^{(1)}+\lambda \hat{\mu} \sigma} S_{x}\right] \Phi^{1}\left[M_{y}+\sigma \frac{\tau}{2} \frac{\hat{\mu}}{c_{0}^{(1)}+\lambda \hat{\mu} \sigma} S_{y}\right]^{T}=\frac{1}{c_{0}^{(1)}+\lambda \hat{\mu} \sigma} F^{0}, \quad n=0,} \\
{\left[M_{x}+\frac{2 \tau}{2 \sigma+1} \frac{\sigma^{2} \hat{\mu}}{c_{0}^{(n+1)}+\lambda \hat{\mu} \sigma} S_{x}\right] \Phi^{n+1}\left[M_{y}+\frac{2 \tau}{2 \sigma+1} \frac{\sigma^{2} \hat{\mu}}{c_{0}^{(n+1)}+\lambda \hat{\mu} \sigma} S_{y}\right]^{T}=\frac{1}{c_{0}^{(n+1)}+\lambda \hat{\mu} \sigma} F^{n}, \quad n \geq 1,}
\end{array}\right.
$$

in which $\Phi^{n}, U^{n}, F^{0}, F^{n} \in \mathbb{R}^{N_{x} \times N_{y}}$ satisfying $\left(\Phi^{n}\right)_{i, j}=\beta_{i j}^{n},\left(U^{n}\right)_{i, j}=\alpha_{i j}^{n}$ and

$$
\left\{\begin{aligned}
F_{i j}^{0}= & \left(c_{0}^{(1)}-\lambda \hat{\mu}(1-\sigma)\right)\left(\phi_{h}^{0}, \varphi_{i} \theta_{j}\right)-\sigma \hat{\mu} \frac{\tau}{2}\left(\nabla \phi_{h}^{0}, \nabla \varphi_{i} \theta_{j}\right)-\hat{\mu}\left(\nabla u_{h}^{0}, \nabla \varphi_{i} \theta_{j}\right)+\hat{\mu}\left(f^{\sigma}, \varphi_{i} \theta_{j}\right) \\
& -\left(\sigma \frac{\tau}{2} \frac{\hat{\mu}}{c_{0}^{(1)}+\lambda \hat{\mu} \sigma}\right)^{2}\left(S_{x} \Phi^{0} S_{y}^{T}\right)_{i j} \\
F_{i j}^{n+1}= & -\sum_{k=0}^{n-1} c_{n-k}^{(n+1)}\left(M_{x} \Phi^{k+1} M_{y}^{T}-M_{x} \Phi^{k} M_{y}^{T}\right)_{i j}+\left(c_{0}^{(n+1)}-\lambda \hat{\mu}(1-\sigma)\right)\left(M_{x} \Phi^{n} M_{y}^{T}\right)_{i j} \\
& +\frac{\sigma(2 \sigma-1) \hat{\mu}}{2 \sigma+1}\left(S_{x} U^{n-1} M_{y}^{T}+M_{x} U^{n-1} S_{y}^{T}\right)_{i j}+\hat{\mu}\left(f^{n+\sigma}, \varphi_{i} \theta_{j}\right) \\
& -2 \sigma(1-\sigma) \hat{\mu} \frac{\tau}{2 \sigma+1}\left(S_{x} \Phi^{n} M_{y}^{T}+M_{x} \Phi^{n} S_{y}^{T}\right)_{i j} \\
& -\left(\frac{4 \sigma^{2} \hat{\mu}}{2 \sigma+1}+(1-\sigma) \hat{\mu}\right)\left(S_{x} U^{n} M_{y}^{T}+M_{x} U^{n} S_{y}^{T}\right)_{i j} \\
& -\sigma^{3}(1-\sigma)\left(\frac{\sigma^{2} \hat{\mu}}{c_{0}^{(n+1)}+\lambda \hat{\mu} \sigma}\right)^{2}\left(S_{x} \Phi^{n} S_{y}^{T}\right)_{i j} .
\end{aligned}\right.
$$

Then the numerical solution $\Phi^{n}$ in (15) can be solved by two steps. For example, for $n=0$, we first solve

$$
\left[M_{x}+\sigma \frac{\tau}{2} \frac{\hat{\mu}}{c_{0}^{(1)}+\lambda \hat{\mu} \sigma} S_{x}\right] \tilde{\Phi}^{1}=\frac{1}{c_{0}^{(1)}+\lambda \hat{\mu} \sigma} F^{0}
$$

then we solve

$$
\left[M_{y}+\sigma \frac{\tau}{2} \frac{\hat{\mu}}{c_{0}^{(1)}+\lambda \hat{\mu} \sigma} S_{y}\right] \Phi^{1}=\left(\tilde{\Phi}^{1}\right)^{T}
$$

\section{Stability and convergence}

Let $X$ be a Banach space with the norm $\|\cdot\|_{X}$. When $v(x, t)$ is defined on the entire time interval $[0, T]$, we define

$$
\begin{aligned}
& C\left([0, T] ; L^{2}(\Omega)\right):=\{v \mid\|v(\cdot, t)\| \in C([0, T])\}, \\
& L^{2}(X):=\left\{v \mid\|v\|_{L^{2}(X)}:=\left(\int_{0}^{T}\|v(\cdot, t)\|_{X}^{2} d t\right)^{\frac{1}{2}}<\infty\right\}, \\
& L^{\infty}(X):=\left\{v \mid\|v\|_{L^{\infty}(X)}:=\sup _{0 \leq t \leq T}\|v(\cdot, t)\|_{X}<\infty\right\} .
\end{aligned}
$$

Let $u_{*}=\Pi_{h}^{1,0} u:[0, T] \rightarrow X_{h}^{r}$ be the $H_{0}^{1}(\Omega)$ projection of the solution $u$ of (1), that is,

$$
\left(\nabla\left(u-u_{*}\right), \nabla \chi\right)=0, \quad \chi \in X_{h}^{r} .
$$


In the following, we study the stability for the ADI Galerkin scheme (14). To this end, we need to introduce some properties of the projection $u_{*}$ and the coefficients $c_{k}^{(n+1)}$ defined by (5).

Lemma 4.1 ([27]) If $\partial^{k} u / \partial t^{k} \in L^{p}\left(H^{r+1}\right)$ for $k=0,1,2$ and $p=2, \infty$, then there exists a constant $C$, independent of $h$, such that

$$
\left\|\frac{\partial^{k}\left(u-u_{*}\right)}{\partial t^{k}}\right\|_{L^{p}\left(H^{j}\right)} \leq C h^{s-j}\left\|\frac{\partial^{k} u}{\partial t^{k}}\right\|_{L^{p}\left(H^{s}\right)},
$$

where $j=0,1,1 \leq s \leq r+1$, and $u_{*}$ is defined in (18).

Lemma 4.2 The following estimates hold:

(a) $c_{0}^{(n+1)}>c_{1}^{(n+1)}>c_{2}^{(n+1)}>\cdots>c_{n}^{(n+1)}>\frac{1-\beta}{2}(n+\sigma)^{-\beta}$;

(b) $\sum_{k=1}^{n-1}\left(c_{k-1}^{(k+1)}-c_{k}^{(k+1)}\right) \leq \frac{4-3 \beta}{2-\beta}(n-1+\sigma)^{1-\beta}$;

(c) $\sum_{k=1}^{n-1} c_{k}^{(k+1)} \leq(n-1+\sigma)^{1-\beta}$.

Proof From the expansion of the coefficients $c_{k}^{(k+1)}$, we can obtain the above estimates. One can also refer to [26] for (a) and [28] for (b) and (c). All this ends the proof.

We also need the following estimates.

Lemma 4.3 ([28]) For any function $u_{h}^{n} \in X_{h}^{r}, n=0,1, \ldots, N$, the following inequalities hold:

$$
\begin{aligned}
& \left(\hat{\delta}_{t} \nabla u_{h}^{n}, \nabla u_{h}^{n+\sigma}\right) \geq \frac{1}{4 \tau}\left(E^{n+1}-E^{n}\right), \quad n \geq 1, \\
& \left(\frac{1}{\hat{\mu}} \sum_{k=0}^{n} c_{k}^{(n+1)}\left(u_{h}^{k+1}-u_{h}^{k}\right), u_{h}^{n+\sigma}\right) \geq \frac{1}{2 \hat{\mu}} \sum_{k=0}^{n} c_{k}^{(n+1)}\left(\left\|u_{h}^{k+1}\right\|^{2}-\left\|u_{h}^{k}\right\|^{2}\right) .
\end{aligned}
$$

Here, $E^{n+1}=(2 \sigma+1)\left\|\nabla u_{h}^{n+1}\right\|^{2}-(2 \sigma-1)\left\|\nabla u_{h}^{n}\right\|^{2}+\left(2 \sigma^{2}+\sigma-1\right)\left\|\nabla u_{h}^{n+1}-\nabla u_{h}^{n}\right\|^{2}$ with $E^{n+1} \geq$ $\frac{1}{\sigma}\left\|\nabla u_{h}^{n+1}\right\|^{2}$.

We are now in the position to present the stability of ADI Galerkin scheme (14).

Theorem 4.1 Suppose that $u_{h}^{n}$ is the solution of ADI Galerkin scheme (14) and $f \in$ $C\left([0, T] ; L^{2}(\Omega)\right)$. Then it holds that

$$
\left\|u_{h}^{m}\right\|_{H_{0}^{1}}^{2} \leq C\left[\left\|\nabla u_{h}^{0}\right\|^{2}+\left\|\phi_{h}^{0}\right\|^{2}+\frac{\tau}{\lambda} \sum_{n=0}^{m-1}\left\|f^{n+\sigma}\right\|^{2}+\tau \sum_{n=1}^{m-1}\left\|\nabla \rho_{h}^{n+\sigma}\right\|^{2}+\tau\left\|\nabla \rho_{h}^{1 / 2}\right\|^{2}\right],
$$

where $\rho_{h}^{1 / 2}$ and $\rho_{h}^{n+\sigma}$ are the perturbations of $\delta_{t} u_{h}^{1 / 2}=\phi_{h}^{1 / 2}+\rho_{h}^{1 / 2}$ and $\hat{\delta}_{t} u_{h}^{n}=\phi_{h}^{n+\sigma}+\rho_{h}^{n+\sigma}$, respectively. 
Proof For $n \geq 1$, let $\chi=\phi_{h}^{n+\sigma}$, or equivalently, $\chi=\hat{\delta}_{t} u_{h}^{n}-\rho_{h}^{n+\sigma}$ in equation (14), some calculations give

$$
\begin{aligned}
& \frac{1}{\hat{\mu}} \sum_{k=0}^{n} c_{n-k}^{(n+1)}\left(\left(\phi_{h}^{k+1}-\phi_{h}^{k}\right), \phi_{h}^{n+\sigma}\right)+\lambda\left\|\phi_{h}^{n+\sigma}\right\|^{2} \\
& \quad+\sigma^{3} \frac{\hat{\mu}}{c_{0}^{(n+1)}+\lambda \hat{\mu} \sigma}\left(\frac{2 \tau}{2 \sigma+1}\right)^{2}\left\|\frac{\partial^{2}}{\partial x \partial y} \phi_{h}^{n+\sigma}\right\|^{2}+\left(\nabla u_{h}^{n+\sigma}, \hat{\delta}_{t} \nabla u_{h}^{n}-\nabla \rho_{h}^{n+\sigma}\right) \\
& \quad=\left(f^{n+\sigma}, \phi_{h}^{n+\sigma}\right) .
\end{aligned}
$$

It follows from Lemma 4.3 that (20) yields

$$
\begin{aligned}
& \frac{1}{2 \hat{\mu}} \sum_{k=0}^{n} c_{n-k}^{(n+1)}\left(\left\|\phi_{h}^{k+1}\right\|^{2}-\left\|\phi_{h}^{k}\right\|^{2}\right)+\lambda\left\|\phi_{h}^{n+\sigma}\right\|^{2} \\
& \quad+\sigma^{3} \frac{\hat{\mu}}{c_{0}^{(n+1)}+\lambda \hat{\mu} \sigma}\left(\frac{2 \tau}{2 \sigma+1}\right)^{2}\left\|\frac{\partial^{2}}{\partial x \partial y} \phi_{h}^{n+\sigma}\right\|^{2}+\frac{1}{4 \tau}\left(E^{n+1}-E^{n}\right) \\
& \leq\left(f^{n+\sigma}, \phi_{h}^{n+\sigma}\right)+\left(\nabla u_{h}^{n+\sigma}, \nabla \rho_{h}^{n+\sigma}\right) .
\end{aligned}
$$

Multiplying $4 \tau$ on both sides of (21), then using the Cauchy-Schwarz inequality for the last two terms on the right-hand side of (21) and removing the nonnegative third term on the left-hand side of (21), we get

$$
\begin{aligned}
& \frac{2 \tau}{\hat{\mu}} \sum_{k=0}^{n} c_{n-k}^{(n+1)}\left(\left\|\phi_{h}^{k+1}\right\|^{2}-\left\|\phi_{h}^{k}\right\|^{2}\right)+4 \lambda \tau\left\|\phi_{h}^{n+\sigma}\right\|^{2}+\left(E^{n+1}-E^{n}\right) \\
& \quad \leq 4 \tau\left\|f^{n+\sigma}\right\|\left\|\phi_{h}^{n+\sigma}\right\|+4 \tau\left\|\nabla u_{h}^{n+\sigma}\right\|\left\|\nabla \rho_{h}^{n+\sigma}\right\| .
\end{aligned}
$$

Summing up $n$ from $n=1$ to $n=m-1$ for (22), we have

$$
\begin{aligned}
& \frac{2 \tau}{\hat{\mu}} \sum_{n=1}^{m-1} \sum_{k=0}^{n} c_{n-k}^{(n+1)}\left(\left\|\phi_{h}^{k+1}\right\|^{2}-\left\|\phi_{h}^{k}\right\|^{2}\right)+4 \lambda \tau \sum_{n=1}^{m-1}\left\|\phi_{h}^{n+\sigma}\right\|^{2}+\left(E^{m}-E^{1}\right) \\
& \leq 4 \tau \sum_{n=1}^{m-1}\left\|f^{n+\sigma}\right\|\left\|\phi_{h}^{n+\sigma}\right\|+4 \tau \sum_{n=1}^{m-1}\left\|\nabla u_{h}^{n+\sigma}\right\|\left\|\nabla \rho_{h}^{n+\sigma}\right\| \\
& \leq 4 \tau \sum_{n=1}^{m-1}\left[\epsilon\left\|f^{n+\sigma}\right\|^{2}+\frac{1}{4 \epsilon}\left\|\phi_{h}^{n+\sigma}\right\|^{2}\right]+4 \tau \sum_{n=1}^{m-1}\left[\frac{1}{4}\left\|\nabla u_{h}^{n+\sigma}\right\|^{2}+\left\|\nabla \rho_{h}^{n+\sigma}\right\|^{2}\right] \\
& \leq \frac{\tau}{\epsilon} \sum_{n=1}^{m-1}\left\|\phi_{h}^{n+\sigma}\right\|^{2}+\tau \sum_{n=1}^{m-1}\left\|\nabla u_{h}^{n+\sigma}\right\|^{2}+4 \tau \epsilon \sum_{n=1}^{m-1}\left\|f^{n+\sigma}\right\|^{2}+4 \tau \sum_{n=1}^{m-1}\left\|\nabla \rho_{h}^{n+\sigma}\right\|^{2} .
\end{aligned}
$$

Here, $\epsilon$ is an arbitrary positive constant which will be given later on. 
Note that

$$
\begin{aligned}
& \frac{2 \tau}{\hat{\mu}} \sum_{n=1}^{m-1} \sum_{k=0}^{n} c_{n-k}^{(n+1)}\left(\left\|\phi_{h}^{k+1}\right\|^{2}-\left\|\phi_{h}^{k}\right\|^{2}\right) \\
& \quad=\frac{2 \tau}{\hat{\mu}} \sum_{n=1}^{m-1}\left[c_{0}^{(n+1)}\left\|\phi_{h}^{n+1}\right\|^{2}-c_{n}^{(n+1)}\left\|\phi_{h}^{0}\right\|^{2}+\sum_{k=1}^{n}\left(c_{n+1-k}^{(n+1)}-c_{n-k}^{(n+1)}\right)\left\|\phi_{h}^{k}\right\|^{2}\right] \\
& \geq-\frac{2}{\Gamma(2-\beta)} T^{1-\beta}\left\|\phi_{h}^{0}\right\|^{2}-\frac{8-6 \beta}{\Gamma(3-\beta)} T^{1-\beta}\left\|\phi_{h}^{1}\right\|^{2}+\frac{2 \tau}{\hat{\mu}} \sum_{k=2}^{m} c_{m-k}^{(m)}\left\|\phi_{h}^{k}\right\|^{2} \\
& \geq-\frac{2}{\Gamma(2-\beta)} T^{1-\beta}\left\|\phi_{h}^{0}\right\|^{2}-\frac{8-6 \beta}{\Gamma(3-\beta)} T^{1-\beta}\left\|\phi_{h}^{1}\right\|^{2}+\frac{\tau}{T^{\beta} \Gamma(1-\beta)} \sum_{k=2}^{m}\left\|\phi_{h}^{k}\right\|^{2},
\end{aligned}
$$

where we have used Lemma 4.2.

So

$$
\begin{aligned}
& \frac{\tau}{T^{\beta} \Gamma(1-\beta)} \sum_{k=1}^{m}\left\|\phi_{h}^{k}\right\|^{2}+4 \lambda \tau \sum_{n=1}^{m-1}\left\|\phi_{h}^{n+\sigma}\right\|^{2}+\left(E^{m}-E^{1}\right) \\
& \leq \frac{2}{\Gamma(2-\beta)} T^{1-\beta}\left\|\phi_{h}^{0}\right\|^{2}+\left(\frac{8-6 \beta}{\Gamma(3-\beta)} T^{1-\beta}+\frac{\tau}{T^{\beta} \Gamma(1-\beta)}\right)\left\|\phi_{h}^{1}\right\|^{2} \\
& \quad+\frac{\tau}{\epsilon} \sum_{n=1}^{m-1}\left\|\phi_{h}^{n+\sigma}\right\|^{2}+\tau \sum_{n=1}^{m-1}\left\|\nabla u_{h}^{n+\sigma}\right\|^{2}+4 \tau \epsilon \sum_{n=1}^{m-1}\left\|f^{n+\sigma}\right\|^{2}+4 \tau \sum_{n=1}^{m-1}\left\|\nabla \rho_{h}^{n+\sigma}\right\|^{2} .
\end{aligned}
$$

Letting $\epsilon=1 /(4 \lambda)$ in (24) and using the estimates for $E^{m}$, we obtain

$$
\begin{aligned}
& \tau \sum_{k=1}^{m}\left\|\phi_{h}^{k}\right\|^{2}+\left\|\nabla u_{h}^{m}\right\|^{2} \\
& \leq C\left[\left\|\nabla u_{h}^{0}\right\|^{2}+\left\|\phi_{h}^{0}\right\|^{2}+\left\|\nabla u_{h}^{1}\right\|^{2}+\left\|\phi_{h}^{1}\right\|^{2}\right. \\
& \left.\quad+\tau \sum_{n=1}^{m-1}\left\|\nabla u_{h}^{n}\right\|^{2}+\frac{\tau}{\lambda} \sum_{n=1}^{m-1}\left\|f^{n+\sigma}\right\|^{2}+\tau \sum_{n=1}^{m-1}\left\|\nabla \rho_{h}^{n+\sigma}\right\|^{2}\right] .
\end{aligned}
$$

Next we estimate the two terms $\left\|\nabla u_{h}^{1}\right\|^{2}$ and $\left\|\phi_{h}^{1}\right\|^{2}$ on the right-hand side of (25), this is the case $n=0$ in (14). Letting $\chi=\phi_{h}^{1 / 2}$, or equivalently, $\chi=\delta_{t} u_{h}^{1 / 2}-\rho_{h}^{1 / 2}$ for the case in (14), we have

$$
\begin{aligned}
& \frac{1}{\hat{\mu}} c_{0}^{(1)}\left(\left(\phi_{h}^{1}-\phi_{h}^{0}\right), \phi_{h}^{1 / 2}\right)+\lambda\left(\phi_{h}^{\sigma}, \phi_{h}^{1 / 2}\right)+2 \frac{\hat{\mu}}{c_{0}^{(1)}+\lambda \hat{\mu} \sigma}\left(\frac{\sigma \tau}{2}\right)^{2}\left\|\frac{\partial^{2}}{\partial x \partial y} \phi_{h}^{1 / 2}\right\|^{2} \\
& \quad+\left(\nabla u_{h}^{\sigma}, \delta_{t} \nabla u_{h}^{1 / 2}-\nabla \rho_{h}^{1 / 2}\right) \\
& =\left(f^{\sigma}, \phi_{h}^{1 / 2}\right) .
\end{aligned}
$$

Multiplying $2 \tau$ on both sides of (26) and removing the nonnegative term $\left\|\partial^{2} \phi_{h}^{1 / 2} / \partial x \partial y\right\|^{2}$, we get

$$
\frac{\tau}{\hat{\mu}} c_{0}^{(1)}\left(\left\|\phi_{h}^{1}\right\|^{2}-\left\|\phi_{h}^{0}\right\|^{2}\right)+2 \tau \lambda\left(\phi_{h}^{\sigma}, \phi_{h}^{1 / 2}\right)+2 \tau\left(\nabla u_{h}^{\sigma}, \delta_{t} \nabla u_{h}^{1 / 2}-\nabla \rho_{h}^{1 / 2}\right) \leq 2 \tau\left(f^{\sigma}, \phi_{h}^{1 / 2}\right) .
$$


Since

$$
2 \tau \lambda\left(\phi_{h}^{\sigma}, \phi_{h}^{1 / 2}\right)=\tau \lambda\left(\sigma\left\|\phi_{h}^{1}\right\|^{2}+(1-\sigma)\left\|\phi_{h}^{0}\right\|^{2}+\left\|\phi_{h}^{0}\right\|\left\|\phi_{h}^{1}\right\|\right) \geq \tau \lambda \sigma\left\|\phi_{h}^{1}\right\|^{2}
$$

and

$$
\begin{aligned}
2 \tau\left(\nabla u_{h}^{\sigma}, \delta_{t} \nabla u_{h}^{1 / 2}\right) & =2\left(\sigma\left\|\nabla u_{h}^{1}\right\|^{2}-(1-\sigma)\left\|\nabla u_{h}^{0}\right\|^{2}+(1-2 \sigma)\left\|\nabla u_{h}^{0}\right\|\left\|\nabla u_{h}^{1}\right\|\right) \\
& \geq\left\|\nabla u_{h}^{1}\right\|^{2}-\left\|\nabla u_{h}^{0}\right\|^{2},
\end{aligned}
$$

where $1-2 \sigma<0$ is used. So, by the Cauchy-Schwarz inequality, equation (27) has the following estimate:

$$
\begin{aligned}
& \frac{\tau}{\hat{\mu}} c_{0}^{(1)}\left(\left\|\phi_{h}^{1}\right\|^{2}-\left\|\phi_{h}^{0}\right\|^{2}\right)+\tau \lambda \sigma\left\|\phi_{h}^{1}\right\|^{2}+\left\|\nabla u_{h}^{1}\right\|^{2}-\left\|\nabla u_{h}^{0}\right\|^{2} \\
& \quad \leq 2 \tau\left\|f^{\sigma}\right\|\left\|\phi_{h}^{1 / 2}\right\|+2 \tau\left\|\nabla u_{h}^{\sigma}\right\|\left\|\nabla \rho_{h}^{\sigma}\right\| \\
& \quad \leq 2 \tau \epsilon\left(\left\|\phi_{h}^{0}\right\|^{2}+\left\|\phi_{h}^{1}\right\|^{2}\right)+\frac{\tau}{4 \epsilon}\left\|f^{\sigma}\right\|^{2}+2 \tau\left(\left\|\nabla u_{h}^{0}\right\|^{2}+\left\|\nabla u_{h}^{1}\right\|^{2}\right)+\frac{\tau}{2}\left\|\nabla \rho_{h}^{1 / 2}\right\|^{2} .
\end{aligned}
$$

Letting $\epsilon=\lambda \sigma / 2$ in (28) and using the equalities $c_{0}^{(1)} \tau / \hat{\mu}=t_{\sigma}^{1-\beta} / \Gamma(2-\beta)$ and $\sigma \tau=t_{\sigma}$, we obtain

$$
\left\|\phi_{h}^{1}\right\|^{2}+\left\|\nabla u_{h}^{1}\right\|^{2} \leq C\left[\left\|\phi_{h}^{0}\right\|^{2}+\left\|\nabla u_{h}^{0}\right\|^{2}+\tau\left\|\nabla u_{h}^{0}\right\|^{2}+\frac{\tau}{\lambda}\left\|f^{\sigma}\right\|^{2}+\tau\left\|\nabla \rho_{h}^{1 / 2}\right\|^{2}\right] .
$$

Combining (25) with (29) gives

$$
\begin{aligned}
\tau \sum_{k=1}^{m}\left\|\phi_{h}^{k}\right\|^{2}+\left\|\nabla u_{h}^{m}\right\|^{2} \leq & C\left[\left\|\nabla u_{h}^{0}\right\|^{2}+\left\|\phi_{h}^{0}\right\|^{2}+\tau \sum_{n=0}^{m-1}\left\|\nabla u_{h}^{n}\right\|^{2}\right. \\
& \left.+\frac{\tau}{\lambda} \sum_{n=0}^{m-1}\left\|f^{n+\sigma}\right\|^{2}+\tau \sum_{n=1}^{m-1}\left\|\nabla \rho_{h}^{n+\sigma}\right\|^{2}+\tau\left\|\nabla \rho_{h}^{1 / 2}\right\|^{2}\right] .
\end{aligned}
$$

From the Gronwall inequality, we readily obtain

$$
\left\|u_{h}^{m}\right\|_{H_{0}^{1}}^{2} \leq C\left[\left\|\nabla u_{h}^{0}\right\|^{2}+\left\|\phi_{h}^{0}\right\|^{2}+\frac{\tau}{\lambda} \sum_{n=0}^{m-1}\left\|f^{n+\sigma}\right\|^{2}+\tau \sum_{n=1}^{m-1}\left\|\nabla \rho_{h}^{n+\sigma}\right\|^{2}+\tau\left\|\nabla \rho_{h}^{1 / 2}\right\|^{2}\right] .
$$

All this completes the proof.

Next we consider the convergence of ADI Galerkin scheme (14).

Theorem 4.2 Let $\left\{u_{h}^{n}\right\}_{n=0}^{N}$ and $u_{*}$ denote the solutions of (14) and (18), respectively. Assume that the solution $u$ in (1) satisfies $u \in L^{\infty}\left(H^{r}\right), \frac{\partial u}{\partial t} \in L^{\infty}\left(H^{r+1}\right), \frac{\partial^{4} u}{\partial t^{4}} \in L^{\infty}\left(L^{2}\right),{ }_{C} D_{0, t}^{\alpha} u \in$ 
$L^{\infty}\left(H^{r}\right), \frac{\partial^{3} u}{\partial x \partial y \partial t} \in L^{\infty}\left(L^{2}\right)$, where $r \geq 1$. Then, for sufficiently small $\tau$,

$$
\begin{aligned}
\max _{0 \leq n \leq N}\left\|u^{n}-u_{h}^{n}\right\|_{H_{0}^{1} \leq} & C\left[h^{r}\left(\|u\|_{L^{\infty}\left(H^{r}\right)}+\left\|\frac{\partial u}{\partial t}\right\|_{L^{2}\left(H^{r+1}\right)}+\frac{1}{\lambda}\left\|_{C} D_{0, t}^{\alpha} u\right\|_{L^{\infty}\left(H^{r}\right)}\right)\right. \\
& +\tau^{2}\left(\left\|\frac{\partial^{3} u}{\partial t^{3}}\right\|_{L^{\infty}\left(L^{2}\right)}+\frac{1}{\lambda}\left\|\frac{\partial^{4} u}{\partial t^{4}}\right\|_{L^{\infty}\left(L^{2}\right)}\right) \\
& \left.+\tau^{\frac{1+\alpha}{2}}\left(\left\|\frac{\partial^{3} u}{\partial x \partial y \partial t}\right\|_{L^{\infty}\left(L^{2}\right)}+h^{r-1}\left\|\frac{\partial u}{\partial t}\right\|_{L^{\infty}\left(H^{r+1}\right)}\right)\right]
\end{aligned}
$$

provided that the initial values $u_{h}^{0}$ and $\varphi_{h}$ satisfy that $\left\|\varphi_{h}-\Pi_{h}^{1,0} \varphi\right\|$ and $\left\|\nabla\left(u_{h}^{0}-\Pi_{h}^{1,0} u_{0}\right)\right\|$ are $O\left(h^{r}\right)$.

Proof Considering the weak form of (8) on the time level $t_{n+\sigma}$ for $n \geq 1$ and $t_{1 / 2}$ for $n=0$, we can derive that

$$
\begin{aligned}
\frac{1}{\hat{\mu}} \sum_{k=0}^{n} c_{n-k}^{n+1}\left(\phi^{k+1}-\phi^{k}, \chi\right)+\lambda\left(\phi\left(x, y, t_{n+\sigma}\right), \chi\right)+\left(\nabla u\left(x, y, t_{n+\sigma}\right), \nabla \chi\right) \\
\quad+\sigma^{3} \frac{\hat{\mu}}{c_{0}^{(n+1)}+\lambda \hat{\mu} \sigma}\left(\frac{2 \tau}{2 \sigma+1}\right)^{2}\left(\frac{\partial^{2}}{\partial x \partial y} \phi^{n+\sigma}, \frac{\partial^{2}}{\partial x \partial y} \chi\right) \\
=\left(R_{1}^{n+\sigma}+R_{2}^{n+\sigma}+f\left(x, y, t_{n+\sigma}\right), \chi\right) \\
\quad+\sigma^{3} \frac{\hat{\mu}}{c_{0}^{(n+1)}+\lambda \hat{\mu} \sigma}\left(\frac{2 \tau}{2 \sigma+1}\right)^{2}\left(\frac{\partial^{2}}{\partial x \partial y} \phi^{n+\sigma}, \frac{\partial^{2}}{\partial x \partial y} \chi\right)
\end{aligned}
$$

with $\delta_{t} u^{1 / 2}=\phi^{1 / 2}+\delta_{t} u^{1 / 2}-\frac{\partial u\left(x, y, t_{1 / 2}\right)}{\partial t}$ for $n=0$ and $\hat{\delta}_{t} u^{n}=\phi^{n+\sigma}+\hat{\delta}_{t} u^{n}-\frac{\partial u\left(x, y, t_{n+\sigma}\right)}{\partial t}$ for $n \geq 1$. Here, $R_{1}^{n+\sigma}$ and $R_{2}^{n+\sigma}$ are the forms as denoted in (4) and (7), respectively, with $\partial^{4} u / \partial t^{4}$ instead of $g^{\prime \prime \prime}(t)$.

Denote $e=u_{*}-u_{h}, \eta=u-u_{*}, \hat{e}=\frac{\partial u_{*}}{\partial t}-\phi_{h}$, and $\hat{\eta}=\phi-\frac{\partial u_{*}}{\partial t}$. Here, $u_{*}$ is defined in (18). Combining (14) with (32), we get the following error equation:

$$
\begin{aligned}
& \frac{1}{\hat{\mu}} \sum_{k=0}^{n} c_{n-k}^{(n+1)}\left(\hat{e}^{k+1}-\hat{e}^{k}, \chi\right)+\lambda\left(\hat{e}^{n+\sigma}, \chi\right)+\left(\nabla e^{n+\sigma}, \nabla \chi\right) \\
& \quad+\sigma^{3} \frac{\hat{\mu}}{c_{0}^{(n+1)}+\lambda \hat{\mu} \sigma}\left(\frac{2 \tau}{2 \sigma+1}\right)^{2}\left(\frac{\partial^{2}}{\partial x \partial y} \hat{e}^{n+\sigma}, \frac{\partial^{2}}{\partial x \partial y} \chi\right) \\
& =\left(\sigma^{n+\sigma}, \chi\right)+\sigma^{3} \frac{\hat{\mu}}{c_{0}^{(n+1)}+\lambda \hat{\mu} \sigma}\left(\frac{2 \tau}{2 \sigma+1}\right)^{2}\left(\kappa^{n+\sigma}, \frac{\partial^{2}}{\partial x \partial y} \chi\right),
\end{aligned}
$$

with $\delta_{t} e^{1 / 2}=\hat{e}^{1 / 2}+\rho^{1 / 2}$ for $n=0$ and $\hat{\delta}_{t} e^{n}=\hat{e}^{n+\sigma}+\rho^{n+\sigma}$ for $n \geq 1$. Here,

$$
\begin{aligned}
\sigma^{n+\sigma} & =-\frac{1}{\hat{\mu}} \sum_{k=0}^{n} c_{n-k}^{(n+1)}\left(\hat{\eta}^{k+1}-\hat{\eta}^{k}\right)-\lambda \hat{\eta}^{n+\sigma}+R_{1}^{n+\sigma}+R_{2}^{n+\sigma}, \\
\kappa^{n+\sigma} & =\frac{\partial^{2}}{\partial x \partial y}\left(\phi_{h}^{n+\sigma}-\hat{\eta}^{n+\sigma}\right), \\
\rho^{1 / 2} & =\hat{\eta}^{1 / 2}+\delta_{t} u^{1 / 2}-\frac{\partial u\left(x, y, t_{1 / 2}\right)}{\partial t}-\delta_{t} \eta^{1 / 2}, \\
\rho^{n+\sigma} & =\hat{\eta}^{n+\sigma}+\hat{\delta}_{t} u^{n}-\frac{\partial u\left(x, y, t_{n+\sigma}\right)}{\partial t}-\hat{\delta}_{t} \eta^{n} .
\end{aligned}
$$


From Theorem 4.1, we can derive the following estimate:

$$
\begin{aligned}
\left\|e^{m}\right\|_{H_{0}^{1}}^{2} \leq & C\left[\left\|\nabla e^{0}\right\|^{2}+\left\|\hat{e}^{0}\right\|^{2}+\frac{\tau}{\lambda} \sum_{n=0}^{m-1}\left\|\sigma^{n+\sigma}\right\|^{2}+\tau \sum_{n=1}^{m-1}\left\|\nabla \rho_{h}^{n+\sigma}\right\|^{2}+\tau\left\|\nabla \rho_{h}^{1 / 2}\right\|^{2}\right. \\
& \left.+\sigma^{3} \frac{\hat{\mu}}{c_{0}^{(n+1)}+\lambda \hat{\mu} \sigma}\left(\frac{2 \tau}{2 \sigma+1}\right)^{2} \tau \sum_{n=0}^{m-1}\left\|\kappa^{n+\sigma}\right\|^{2}\right] .
\end{aligned}
$$

Using the triangle inequality and (34), we have

$$
\begin{aligned}
\left\|u^{m}-u_{h}^{m}\right\|_{H_{0}^{1}}^{2} \leq & C\left[\left\|\eta^{m}\right\|_{H_{0}^{1}}^{2}+\left\|\nabla e^{0}\right\|^{2}+\left\|\hat{e}^{0}\right\|^{2}+\frac{\tau}{\lambda} \sum_{n=0}^{m-1}\left\|\sigma^{n+\sigma}\right\|^{2}+\tau \sum_{n=1}^{m-1}\left\|\nabla \rho_{h}^{n+\sigma}\right\|^{2}\right. \\
& \left.+\tau\left\|\nabla \rho_{h}^{1 / 2}\right\|^{2}+\sigma^{3} \frac{\hat{\mu}}{c_{0}^{(n+1)}+\lambda \hat{\mu} \sigma}\left(\frac{2 \tau}{2 \sigma+1}\right)^{2} \tau \sum_{n=0}^{m-1}\left\|\kappa^{n+\sigma}\right\|^{2}\right] .
\end{aligned}
$$

So it remains to estimate the terms on the right-hand side of (35). Firstly, by Lemma 4.1, we obtain

$$
\left\|\eta^{n}\right\|_{H_{0}^{1}}^{2} \leq\|\eta\|_{L^{\infty}\left(H^{1}\right)}^{2} \leq C h^{2 r}\|u\|_{L^{\infty}\left(H^{r+1}\right)}^{2} .
$$

Since

$$
\frac{\tau}{\lambda} \sum_{n=0}^{m-1}\left\|-\frac{1}{\mu} \sum_{k=0}^{n} c_{n-k}^{(n+1)}\left(\hat{\eta}^{k+1}-\hat{\eta}^{k}\right)\right\|^{2} \leq C \frac{1}{\lambda}\left\|_{C} D_{0, t}^{\alpha} \eta\right\|_{L^{\infty}\left(L^{2}\right)}^{2} \leq C \frac{1}{\lambda} h^{2 r}\left\|_{C} D_{0, t}^{\alpha} u\right\|_{L^{\infty}\left(H^{r}\right)}^{2},
$$

one has

$$
\begin{aligned}
\frac{\tau}{\lambda} \sum_{n=0}^{m-1}\left\|\sigma^{n+\sigma}\right\|^{2}= & \frac{\tau}{\lambda} \sum_{n=0}^{m-1}\left[\left\|-\frac{1}{\mu} \sum_{k=0}^{n} c_{n-k}^{(n+1)}\left(\hat{\eta}^{k+1}-\hat{\eta}^{k}\right)\right\|^{2}\right. \\
& \left.+\lambda^{2}\left\|\hat{\eta}^{n+\sigma}\right\|^{2}+\left\|R_{1}^{n+\sigma}+R_{2}^{n+\sigma}\right\|^{2}\right] \\
\leq & C\left[\frac{1}{\lambda} h^{2 r}\left\|_{C} D_{0, t^{\alpha}}^{\alpha}\right\|_{L^{\infty}\left(H^{r}\right)}^{2}\right. \\
& \left.+\lambda h^{2 r}\left\|\frac{\partial u}{\partial t}\right\|_{L^{2}\left(H^{r}\right)}^{2}+\frac{1}{\lambda} \tau^{4}\left\|\frac{\partial^{4} u}{\partial t^{4}}\right\|_{L^{\infty}\left(L^{2}\right)}^{2}\right] .
\end{aligned}
$$

For the fifth term on the right-hand side of (35), we have

$$
\begin{aligned}
\tau \sum_{n=1}^{m-1}\left\|\nabla \rho_{h}^{n+\sigma}\right\|^{2} & \leq \tau \sum_{n=1}^{m-1}\left\|\nabla \hat{\eta}^{n+\sigma}\right\|^{2}+\tau \sum_{n=1}^{m-1}\left\|\hat{\delta}_{t} u^{n}-\frac{\partial u\left(x, y, t_{n+\sigma}\right)}{\partial t}\right\|^{2}+\tau \sum_{n=1}^{m-1}\left\|\hat{\delta}_{t} \nabla \eta^{n}\right\|^{2} \\
& \leq C\left[h^{2 r}\left\|\frac{\partial u}{\partial t}\right\|_{L^{2}\left(H^{r+1}\right)}^{2}+\tau^{4}\left\|\frac{\partial^{3} u}{\partial t^{3}}\right\|_{L^{\infty}\left(L^{2}\right)}^{2}\right]
\end{aligned}
$$


where we have used the following inequality:

$$
\left\|\hat{\delta}_{t} \eta^{n}\right\|^{2} \leq C\left[\left\|\frac{\eta^{n+1}-\eta^{n}}{\tau}\right\|^{2}+\left\|\frac{\eta^{n}-\eta^{n-1}}{\tau}\right\|^{2}+\left\|\frac{\eta^{n+1}-\eta^{n-1}}{2 \tau}\right\|^{2}\right] .
$$

Similarly, we can obtain

$$
\begin{aligned}
\left\|\nabla \rho_{h}^{1 / 2}\right\|^{2} & \leq \tau\left\|\nabla \hat{\eta}^{1 / 2}\right\|^{2}+\tau\left\|\delta_{t} u^{1 / 2}-\frac{\partial u\left(x, y, t_{1 / 2}\right)}{\partial t}\right\|^{2}+\tau\left\|\delta_{t} \nabla \eta^{1 / 2}\right\|^{2} \\
& \leq C\left[h^{2 r}\left\|\frac{\partial u}{\partial t}\right\|_{L^{2}\left(H^{r+1}\right)}^{2}+\tau^{4}\left\|\frac{\partial^{3} u}{\partial t^{3}}\right\|_{L^{\infty}\left(L^{2}\right)}^{2}\right] .
\end{aligned}
$$

For the last term on the right-hand side of equation (35), we have the estimate below (cf. [27, p.1275]).

$$
\begin{aligned}
& \sigma^{3} \frac{\hat{\mu}}{c_{0}^{(n+1)}+\lambda \hat{\mu} \sigma}\left(\frac{2 \tau}{2 \sigma+1}\right)^{2} \tau \sum_{n=0}^{m-1}\left\|\kappa^{n+\sigma}\right\|^{2} \\
& \leq C \tau^{1+\alpha} \tau \sum_{n=0}^{m-1}\left[\left\|\frac{\partial^{2} \phi_{h}^{n+\sigma}}{\partial x \partial y}\right\|^{2}+\left\|\frac{\partial^{2} \hat{\eta}^{n+\sigma}}{\partial x \partial y}\right\|^{2}\right] \\
& \quad \leq C \tau^{1+\alpha} \tau \sum_{n=0}^{m-1}\left[\left\|\frac{\partial^{3} u}{\partial x \partial y \partial t}\right\|_{L^{\infty}\left(L^{2}\right)}^{2}+\left\|\frac{\partial^{2} \hat{\eta}}{\partial x \partial y}\right\|_{L^{\infty}\left(L^{2}\right)}^{2}\right] \\
& \quad \leq C \tau^{1+\alpha}\left[\left\|\frac{\partial^{3} u}{\partial x \partial y \partial t}\right\|_{L^{\infty}\left(L^{2}\right)}^{2}+h^{2 r-2}\left\|\frac{\partial u}{\partial t}\right\|_{L^{\infty}\left(H^{r+1}\right)}^{2}\right] .
\end{aligned}
$$

So, combining the above estimate (36)-(40) for (35), we obtain

$$
\begin{aligned}
\max _{0 \leq n \leq N}\left\|u^{n}-u_{h}^{n}\right\|_{H_{0}^{1}} \leq & C\left[h^{r}\left(\|u\|_{L^{\infty}\left(H^{r}\right)}+\left\|\frac{\partial u}{\partial t}\right\|_{L^{2}\left(H^{r+1}\right)}+\frac{1}{\lambda}\left\|_{C} D_{0, t}^{\alpha} u\right\|_{L^{\infty}\left(H^{r}\right)}\right)\right. \\
& +\tau^{2}\left(\left\|\frac{\partial^{3} u}{\partial t^{3}}\right\|_{L^{\infty}\left(L^{2}\right)}+\frac{1}{\lambda}\left\|\frac{\partial^{4} u}{\partial t^{4}}\right\|_{L^{\infty}\left(L^{2}\right)}\right) \\
& \left.+\tau^{\frac{1+\alpha}{2}}\left(\left\|\frac{\partial^{3} u}{\partial x \partial y \partial t}\right\|_{L^{\infty}\left(L^{2}\right)}+h^{r-1}\left\|\frac{\partial u}{\partial t}\right\|_{L^{\infty}\left(H^{r+1}\right)}\right)\right],
\end{aligned}
$$

provided that $u_{h}^{0}$ and $\varphi_{h}$ are chosen such that $\left\|\varphi_{h}-\Pi_{h}^{1,0} \varphi\right\|$ and $\left\|\nabla e^{0}\right\|$ are $O\left(h^{r}\right)$. This completes the proof.

Remark 4.1 It seems that the temporal convergent order would be destroyed due to the presence of the term $O\left(\tau^{(1+\alpha) / 2}\right)$ in Theorem 4.2. However, in the practical computation, the convergence order in time always be two for (14). The disagreement probably means that the theoretical analyses may be improved. In fact, the term $O\left(\tau^{(1+\alpha) / 2}\right)$ comes from the error estimate of the accumulation for adding small term at a given time level. One possible way to improve the result is by introducing a much smaller disturbance term to construct the ADI Galerkin scheme; however, it seems that the resulting scheme does not improve the numerical results in practical computation but only causes more complicated computation. 


\section{Numerical examples}

Here we present numerical examples to verify the theoretical analysis of ADI Galerkin scheme (14).

Example 5.1 Consider the following two-dimensional time-fractional partial differential equation with damping:

$$
\left\{\begin{array}{l}
{ }_{C} D_{0, t}^{\alpha} u+\frac{\partial u}{\partial t}=\Delta u+f(x, y, t), \quad(x, y, t) \in(0, \pi) \times(0, \pi) \times(0,1], \\
\left.u\right|_{\partial \Omega}=0, \quad u(x, y, 0)=\sin (x) \sin (y),\left.\quad \frac{\partial u}{\partial t}\right|_{t=0}=\sin (x) \sin (y) .
\end{array}\right.
$$

The right-hand side function $f$ is chosen such that the exact solution to (42) is

$$
u(x, y, t)=\left(t^{4}+t+1\right) \sin (x) \sin (y) .
$$

We use the cubic element in this example. We first test the global maximum $H_{0}^{1}$-error $\max _{0 \leq n \leq N}\left\|u^{n}-u_{h}^{n}\right\|_{H_{0}^{1}}$ with respect to time by fixing the spatial step $h=\pi / 256$. The numerical results are presented in Table 1. From Table 1, we observe that the temporal convergence orders are two, which is in good agreement with the theoretical analysis.

Letting $\tau=1 / 4,096$, the numerical results for (42) are then demonstrated in Table 2 . Recall that the number $r$ in Theorem 4.2 is referred to as the degree of polynomial in the family $\left\{X_{h}^{r}\right\}$, and the piecewise cubic functions in a plane domain correspond to $r=3$ [29]. So we expect that the convergence order in space for the ADI Galerkin scheme (14) is third-order accurate according to Theorem 4.2. From Table 2, we indeed observe the desired result and verify the theoretical analysis given in Theorem 4.2.

Next, we compare the Galerkin ADI scheme (14) with the ADI finite difference scheme (denoted by L1ADIFD), where the Caputo derivative in time is discretized by the L1 method [24].

Table 1 The global maximum $H_{0}^{1}$-errors $\max _{0 \leq n \leq N}\left\|u^{n}-u_{h}^{n}\right\|_{H_{0}^{1}}$ for equation (42) with $h=\pi / 256$

\begin{tabular}{llllll}
\hline $\boldsymbol{\alpha}$ & $\boldsymbol{N}$ & & & & \\
\cline { 2 - 6 } & $\mathbf{8}$ & $\mathbf{1 6}$ & $\mathbf{3 2}$ & $\mathbf{6 4}$ & $\mathbf{1 2 8}$ \\
\hline 1.35 & $5.071 \mathrm{E}-02$ & $1.391 \mathrm{E}-02$ & $3.650 \mathrm{E}-03$ & $9.374 \mathrm{E}-04$ & $2.381 \mathrm{E}-04$ \\
& - & 1.8661 & 1.9303 & 1.9612 & 1.9771 \\
1.55 & $3.522 \mathrm{E}-02$ & $9.681 \mathrm{E}-03$ & $2.550 \mathrm{E}-03$ & $6.578 \mathrm{E}-04$ & $1.677 \mathrm{E}-04$ \\
& - & 1.8631 & 1.9245 & 1.9549 & 1.9715 \\
1.75 & $1.992 \mathrm{E}-02$ & $5.513 \mathrm{E}-03$ & $1.466 \mathrm{E}-03$ & $3.825 \mathrm{E}-04$ & $9.864 \mathrm{E}-05$ \\
& - & 1.8535 & 1.9105 & 1.9388 & 1.9551 \\
\hline
\end{tabular}

Table 2 The global maximum $H_{0}^{1}$-errors $\max _{0 \leq n \leq N}\left\|u^{n}-u_{h}^{n}\right\|_{H_{0}^{1}}$ for equation (42) with $\tau=1 / 4,096$

\begin{tabular}{llll}
\hline $\boldsymbol{\alpha}$ & $\boldsymbol{h}$ & & \\
\cline { 2 - 4 } & $\boldsymbol{\pi / 4}$ & $\boldsymbol{\pi / 8}$ & $\boldsymbol{\pi / 1 6}$ \\
\hline 1.35 & $9.485 \mathrm{E}-04$ & $1.181 \mathrm{E}-04$ & $1.550 \mathrm{E}-05$ \\
& - & 3.0056 & 2.9301 \\
1.55 & $9.443 \mathrm{E}-04$ & $1.180 \mathrm{E}-04$ & $1.511 \mathrm{E}-05$ \\
& - & 3.0010 & 2.9650 \\
1.75 & $9.450 \mathrm{E}-04$ & $1.178 \mathrm{E}-04$ & $1.485 \mathrm{E}-05$ \\
& - & 3.0041 & 2.9875 \\
\hline
\end{tabular}


Table 3 Comparison of the $L^{\infty}$ errors $\max _{0 \leq n \leq N}\left\|u_{h}^{n}-u^{n}\right\|_{\infty}$ with $h=\pi / 200$

\begin{tabular}{rrrlll}
\hline $\boldsymbol{\alpha}$ & $\mathbf{1 / \boldsymbol { \tau }}$ & Error [24] & Cvge & Error (14) & Cvge \\
\hline 1.3 & 10 & $1.897 \mathrm{E}-02$ & - & $2.029 \mathrm{E}-02$ & - \\
& 20 & $5.674 \mathrm{E}-03$ & 1.7413 & $5.487 \mathrm{E}-03$ & 1.8868 \\
& 40 & $1.702 \mathrm{E}-03$ & 1.7372 & $1.427 \mathrm{E}-03$ & 1.9434 \\
& 80 & $5.133 \mathrm{E}-04$ & 1.7293 & $3.626 \mathrm{E}-04$ & 1.9763 \\
& 160 & $1.566 \mathrm{E}-04$ & 1.7131 & $8.981 \mathrm{E}-05$ & 2.0133 \\
1.5 & $3.911 \mathrm{E}-02$ & - & $1.659 \mathrm{E}-02$ & - \\
& 10 & $1.356 \mathrm{E}-02$ & 1.5286 & $4.509 \mathrm{E}-03$ & 1.8792 \\
& 20 & $4.720 \mathrm{E}-03$ & 1.5221 & $1.179 \mathrm{E}-03$ & 1.9358 \\
& 40 & $1.650 \mathrm{E}-03$ & 1.5164 & $3.010 \mathrm{E}-04$ & 1.9692 \\
& $8.792 \mathrm{E}-04$ & 1.5102 & $7.496 \mathrm{E}-05$ & 2.0055 \\
1.7 & $7.908 \mathrm{E}-02$ & - & $1.202 \mathrm{E}-02$ & - \\
& 160 & $3.162 \mathrm{E}-02$ & 1.3225 & $3.283 \mathrm{E}-03$ & 1.8718 \\
& $1.274 \mathrm{E}-02$ & 1.3120 & $8.648 \mathrm{E}-04$ & 1.9245 \\
& 10 & $5.148 \mathrm{E}-03$ & 1.3068 & $2.229 \mathrm{E}-04$ & 1.9561 \\
& 20 & $2.085 \mathrm{E}-03$ & 1.3037 & $5.604 \mathrm{E}-05$ & 1.9918 \\
\hline & & & & &
\end{tabular}

Example 5.2 In [24], the coefficient $\lambda$ in (1) is $\lambda=1$ and the exact solution is

$$
u(x, y, t)=\sin (x) \sin (y) t^{3+\alpha}, \quad(x, y, t) \in(0, \pi) \times(0, \pi) \times(0,1] .
$$

The accuracy of the L1ADIFD scheme is $O\left(\tau^{3-\alpha}+h^{2}\right)$. In this example, we use the linear element and define the $L^{\infty}$ error on the grid points $\left\{x_{i}, y_{j}\right\}$ at $t=t_{n}$ by $\left\|u^{n}-u_{h}^{n}\right\|_{\infty}=$ $\max _{1 \leq i, j \leq N_{x}}\left|u\left(x_{i}, y_{j}, t_{n}\right)-u_{h}^{n}\left(x_{i}, y_{j}\right)\right|$. The numerical results are demonstrated in Table 3 . Obviously, the Galerkin ADI scheme (14) shows better performances than the L1ADIFD scheme, especially when $\alpha$ increases. It is verified again that the accuracy of ADI Galerkin scheme (14) in time is of order two.

\section{Conclusion}

In this paper, by using the $\mathrm{L} 2-1_{\sigma}$ method for the Caputo derivative and the ADI finite element method for the spatial derivative, we obtain the efficient ADI Galerkin scheme (14) for solving (1). The corresponding theoretical analyses, such as stability and error estimate, are also presented. We remark that the proposed method is derived on the direct discretization for the Caputo derivative at the non-grid point in time. Thus this leads to the significant difference with those existing numerical methods, such as [15].

Obviously, the present method can be extended to three-dimensional problems. The corresponding theoretical analyses are similar to that of the two-dimensional case. In the future work, we would combine the L2-1 $\sigma$ method with non-uniform meshes technique [30] to deal with the nonsmooth solution issue.

\section{Funding}

The work is supported by the National Natural Science Foundation of China [grant number 11671251], the Doctoral Starting up Foundation of Guilin University of Technology [grant number 002401003551].

Competing interests

The authors declare that they have no competing interests.

Authors' contributions

All authors contributed equally and significantly in this manuscript, and they read and approved the final manuscript.

Author details

${ }^{1}$ College of Science, Guilin University of Technology, 12 Jiangan Road, Guilin, Guangxi 541004, China. ${ }^{2}$ Department of Mathematics, Shanghai University, 99 Shangda Road, BaoShan District, Shanghai, 200444, China. 


\section{Publisher's Note}

Springer Nature remains neutral with regard to jurisdictional claims in published maps and institutional affiliations.

\section{Received: 22 July 2017 Accepted: 27 October 2017 Published online: 09 November 2017}

\section{References}

1. Singh, J, Kumar, D, Qurashi, MA, Baleanu, D: Analysis of a new fractional model for damped Bergers' equation. Open Phys. 15, 35-41 (2017)

2. Zeng, S, Baleanu, D, Bai, Y, Wu, G: Fractional differential equations of Caputo-Katugampola type and numerical solutions. Appl. Math. Comput. 315, 549-554 (2017)

3. Metzler, R, Klafter, J: The random walk's guide to anomalous diffusion: a fractional dynamics approach. Phys. Rep. $339(1), 1-77(2000)$

4. Solomon, TH, Weeks, ER, Swinney, HL: Observation of anomalous diffusion and Lévy flights in a two-dimensional rotating flow. Phys. Rev. Lett. 71(24), 3975-3978 (1993)

5. Povstenko, YZ: Fractional Cattaneo-type equations and generalized thermoelasticity. J. Therm. Stresses 34(2), 97-114 (2011)

6. Brunner, $\mathrm{H}, \mathrm{Han}, \mathrm{H}$, Yin, D: Artificial boundary conditions and finite difference approximations for a time-fractiona diffusion-wave equation on a two-dimensional unbounded spatial domain. J. Comput. Phys. 276, 541-562 (2014)

7. Chen, A, Li, CP: Numerical solution of fractional diffusion-wave equation. Numer. Funct. Anal. Optim. 37(1), 19-39 (2016)

8. Huang, J, Tang, Y, Vázquez, L, Yang, J: Two finite difference schemes for time fractional diffusion-wave equation. Numer. Algorithms 64(4), 707-720 (2013)

9. Wang, Y: A compact finite difference method for a class of time fractional convection-diffusion-wave equations with variable coefficients. Numer. Algorithms 70(3), 625-651 (2015)

10. Zhuang, P, Liu, F, Anh, V, Turner, I: New solution and analytical techniques of the implicit numerical method for the anomalous subdiffusion equation. SIAM J. Numer. Anal. 46(2), 1079-1095 (2008)

11. Chen, $A, L i, C P: A$ novel compact ADI scheme for the time-fractional subdiffusion equation in two space dimensions. Int. J. Comput. Math. 93(6), 889-914 (2016)

12. Hu, Y, Li, CP, Li, HF: The finite difference method for Caputo-type parabolic equation with fractional Laplacian: one-dimensional case. Chaos Solitons Fractals 102, 361-371 (2017)

13. Yaseen, M, Abbas, M, Nazir, T, Baleanu, D: A finite difference scheme based on cubic trigonometric B-splines for a time fractional diffusion-wave equation. Adv. Differ. Equ. 2017, Article ID 274 (2017)

14. Jin, B, Lazarov, R, Zhou, Z: Error estimates for a semidiscrete finite element method for fractional order parabolic equations. SIAM J. Numer. Anal. 51(1), 445-466 (2013)

15. Li, L, Xu, D, Luo, M: Alternating direction implicit Galerkin finite element method for the two-dimensional fractional diffusion-wave equation. J. Comput. Phys. 255, 471-485 (2013)

16. Mustapha, $K$, Abdallah, B, Furati, KM: A discontinuous Petrov-Galerkin method for time-fractional diffusion equations. SIAM J. Numer. Anal. 52(5), 2512-2529 (2014)

17. Zeng, FH, Li, CP, Liu, F: High-order explicit-implicit numerical methods for nonlinear anomalous diffusion equations. Eur. Phys. J. Spec. Top. 222(8), 1885-1900 (2013)

18. Zeng, FH, Li, CP, Liu, F, Turner, I: Numerical algorithms for time-fractional subdiffusion equation with second-order accuracy. SIAM J. Sci. Comput. 37(1), 55-78 (2015)

19. Chen, F, Xu, Q, Hesthaven, JS: A multi-domain spectral method for time-fractional differential equations. J. Comput. Phys. 293, 157-172 (2015)

20. Li, X, Xu, CJ: A space-time spectral method for the time fractional diffusion equation. SIAM J. Numer. Anal. 47(3), 2108-2131 (2009)

21. Li, CP, Zeng, FH: Numerical Methods for Fractional Calculus, vol. 24. Chapman \& Hall/CRC Press, Boca Raton (2015)

22. Li, CP, Chen, A: Numerical methods for fractional partial differential equations. Int. J. Comput. Math. (2017). doi:10.1080/00207160.2017.1343941

23. Chen, J, Liu, F, Anh, V, Shen, S, Liu, Q, Liao, C: The analytical solution and numerical solution of the fractional diffusion-wave equation with damping. Appl. Math. Comput. 219(4), 1737-1748 (2012)

24. Ren, J, Gao, G: Efficient and stable numerical methods for the two-dimensional fractional Cattaneo equation. Numer. Algorithms 69(4), 795-818 (2015)

25. Zhao, X, Sun, Z: Compact Crank-Nicolson schemes for a class of fractional Cattaneo equation in inhomogeneous medium. J. Sci. Comput. 62(3), 747-771 (2015)

26. Alikhanov, AA: A new difference scheme for the time fractional diffusion equation. J. Comput. Phys. 280, 424-438 (2015)

27. Fernandes, Rl, Fairweather, G: An alternating direction Galerkin method for a class of second-order hyperbolic equations in two space variables. SIAM J. Numer. Anal. 28(5), 1265-1281 (1991)

28. Sun, H, Sun, Z, Gao, G: Some temporal second order difference schemes for fractional wave equations. Numer. Methods Partial Differ. Equ. 32, 970-1001 (2016)

29. Thomée, V: Galerkin Finite Element Methods for Parabolic Problems. Springer, Berlin (2006)

30. Li, CP, Yi, Q, Chen, A: Finite difference methods with non-uniform meshes for nonlinear fractional differential equations. J. Comput. Phys. 316, 614-631 (2016) 